\title{
Local structure supports learning of deterministic behavior in recurrent neural networks
}

\author{
Jonathan Binas ${ }^{1,2^{*}}$, Giacomo Indiveri ${ }^{1,2}$, Michael Pfeiffer ${ }^{1,2}$ \\ From 24th Annual Computational Neuroscience Meeting: CNS*2015 \\ Prague, Czech Republic. 18-23 July 2015
}

Many aspects of behavior, such as language, navigation, or logical reasoning require strongly deterministic and sequential processing of sensory and internal signals. This type of computation can be modeled conveniently in the framework of finite automata.

In this study, we present a recurrent neural network based on biologically plausible circuit motifs, which is able to learn such deterministic behavior from sensory input and reinforcement signals. We find that simple, biologically plausible structural constraints lead to optimized solutions and significantly improve the training process.

Previous work $[1,2]$ has shown how arbitrary finite automata can be hand-crafted in simple networks of

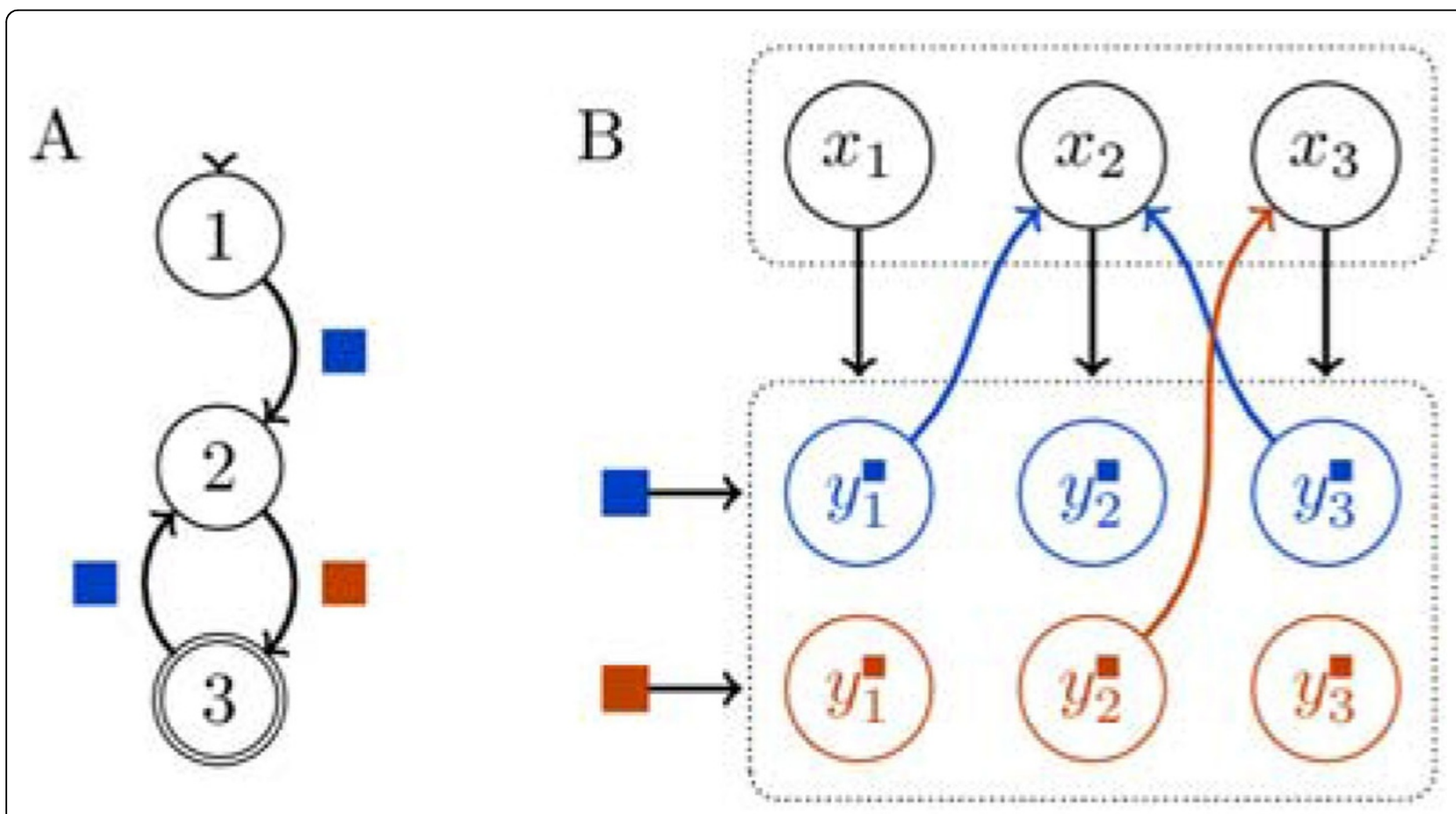

Figure 1 Abstract graphical representation of an example finite automaton (A) and corresponding neural implementation (B). The dotted boxes represent Winner-Take-All circuits in which only one population is active at a time. The populations labeled $x$ represent the states while the populations labeled $y$ implement the conditional transitions between states. The colored connections are learned during training.

\footnotetext{
*Correspondence: jbinas@ini.ethz.ch

'Institute of Neuroinformatics, University of Zurich, Zurich, Switzerland

Full list of author information is available at the end of the article
} 
neural populations by interconnecting multiple WinnerTake-All units - small circuit motifs that match the properties of cortical canonical microcircuits [3,4]. Figure 1 illustrates this transformation from an automaton to neural network with populations of neurons encoding either the state or potential state transitions. We extend that work by introducing a reinforcement learning mechanism whose weight updates take the form of reward-modulated Hebbian rule. This mechanism leads to reconfiguration of the network connectivity in such a way that a desired behavior is learned from sequences of inputs and reward signals.

As a key result of our study, we find that simple constraints on the network topology, favoring local connectivity patterns, lead to dramatic improvements both in training time and in the optimality of the found solution, where the optimum is defined as the automaton with the minimum number of states used to implement a given behavior. These structural constraints correspond well to biological neural systems, where short-range connections far outnumber long-range ones.

\footnotetext{
Authors' details

${ }^{1}$ Institute of Neuroinformatics, University of Zurich, Zurich, Switzerland. ${ }^{2}$ ETH Zurich, Zurich, Switzerland.
}

Published: 18 December 2015

\section{References}

1. Rutishauser U, Douglas RJ: State-dependent computation using coupled recurrent networks. Neural Comput 2009, 21(2):478-509.

2. Neftci E, Binas J, Rutishauser U, Chicca E, Indiveri G, Douglas JR: Synthesizing cognition in neuromorphic electronic systems. Proc Natl Acad Sci U S A 2013, 110(37):3468-3476.

3. Douglas RJ, Martin KAC: Neuronal circuits of the neocortex. Annu Rev Neurosci 2004, 27(1):419-451.

4. Douglas RJ, Martin KAC: Recurrent neuronal circuits in the neocortex. Curr Biol 2007, 17(13):496-500.

doi:10.1186/1471-2202-16-S1-P195

Cite this article as: Binas et al:: Local structure supports learning of deterministic behavior in recurrent neural networks. BMC Neuroscience 2015 16(Suppl 1):P195.

\section{Submit your next manuscript to BioMed Central} and take full advantage of:

- Convenient online submission

- Thorough peer review

- No space constraints or color figure charges

- Immediate publication on acceptance

- Inclusion in PubMed, CAS, Scopus and Google Scholar

- Research which is freely available for redistribution

Submit your manuscript at www.biomedcentral.com/submit
C Biomed Central 